Canadian Journal of Applied Linguistics

Revue canadienne de linguistique appliquée

\title{
Étude de la perception des apprenants de français langue étrangère concernant l'enseignement des langues basé sur les tâches
}

\section{Caroline Payant}

Volume 22, Number 1, 2019

Special Issue: In Memory of Larry Vandergrift

Numéro spécial : à la mémoire de Larry Vandergrift

URI: https://id.erudit.org/iderudit/1060904ar

DOI: https://doi.org/10.7202/1060904ar

See table of contents

Publisher(s)

University of New Brunswick

ISSN

1920-1818 (digital)

Explore this journal

Cite this article

Payant, C. (2019). Étude de la perception des apprenants de français langue étrangère concernant l'enseignement des langues basé sur les tâches. Canadian Journal of Applied Linguistics / Revue canadienne de linguistique appliquée, 22(1). https://doi.org/10.7202/1060904ar

\section{Article abstract}

Research on task-based language teaching (TBLT) shows that second and foreign language learners develop their language skills through learner-learner interactions that take place when performing pedagogical tasks (Bygate, 2015; Kim, 2017). To date, a significant number of researchers have focused on task characteristics that promote interaction, on task implementation, as well as on teachers' perceptions of TBLT (East, 2012; Hu, 2013; McDonough \&amp; Chaikitmongkol, 2007). Despite the enthusiasm surrounding TBLT, learners' perspectives on this approach remain underexplored. Consequently, the present study examined the perceptions of 12 learners of French as a foreign language with regard to TBLT in general, as well as their perceptions of four types of pedagogical tasks (Ellis, 2003). The qualitative analysis of data collected via interviews with each learner shows that they benefited from participating in task-based interaction and highlights task characteristics that should be taken into account when creating pedagogical tasks.
Copyright (c) Caroline Payant, 2019

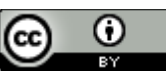

This document is protected by copyright law. Use of the services of Érudit (including reproduction) is subject to its terms and conditions, which can be viewed online.

https://apropos.erudit.org/en/users/policy-on-use/ 


\title{
Étude de la perception des apprenants de français langue étrangère concernant l'enseignement des langues basé sur les tâches
}

\author{
Caroline Payant \\ Université du Québec à Montréal
}

\begin{abstract}
Résumé
La recherche portant sur l'enseignement des langues basé sur les tâches (ELBT) montre que les apprenants de langue seconde ou étrangère développent leurs compétences langagières grâce aux interactions ayant lieu lors de la réalisation de tâches avec leurs pairs (Bygate, 2015 ; Kim, 2017). Pour cette raison, un nombre important de chercheurs se sont intéressés aux caractéristiques des tâches qui favorisent l'interaction, ainsi qu'à la façon optimale d'exécuter ces dernières et aux perceptions des enseignants concernant cette approche basée sur les tâches (East, 2012 ; Hu, 2013 ; McDonough et Chaikitmongkol, 2007). Cependant, malgré l'enthousiasme qui entoure l'ELBT, le point de vue des apprenants sur cette approche demeure peu documenté. La présente étude a donc examiné les perceptions qu'avaient 12 apprenants de français langue étrangère à propos de l'ELBT en général, ainsi que leurs perceptions de quatre types de tâches langagières spécifiques (Ellis, 2003). L'analyse qualitative des données, lesquelles ont été recueillies par le biais d'entrevues, montre que ces apprenants ont apprécié les tâches et, elle permet de mettre en lumière les caractéristiques devant être prises en compte lors de la création des tâches.
\end{abstract}

\begin{abstract}
Research on task-based language teaching (TBLT) shows that second and foreign language learners develop their language skills through learner-learner interactions that take place when performing pedagogical tasks (Bygate, 2015; Kim, 2017). To date, a significant number of researchers have focused on task characteristics that promote interaction, on task implementation, as well as on teachers' perceptions of TBLT (East, 2012; Hu, 2013; McDonough \& Chaikitmongkol, 2007). Despite the enthusiasm surrounding TBLT, learners' perspectives on this approach remain underexplored. Consequently, the present study examined the perceptions of 12 learners of French as a foreign language with regard to TBLT in general, as well as their perceptions of four types of pedagogical tasks (Ellis, 2003). The qualitative analysis of data collected via interviews with each learner shows that they benefited from participating in task-based interaction and highlights task characteristics that should be taken into account when creating pedagogical tasks.
\end{abstract}




\section{Étude de la perception des apprenants de français langue étrangère concernant l'enseignement des langues basé sur les tâches}

\section{Introduction}

Les études portant sur l'enseignement des langues basé sur les tâches (ELBT) démontrent que les apprenants de langue seconde (L2) ou étrangère arrivent à développer leurs compétences langagières grâce aux interactions ayant lieu lors de l'exécution de tâches avec leurs pairs (Bygate, 2015 ; Gass et Mackey, 2007 ; Kim, 2015 ; Van den Branden, 2016). En s'appuyant sur les principes de la théorie interactionnelle (Ellis, 2017 ; Gass, 2015), les chercheurs soutiennent en effet que le travail effectué par le biais de tâches collaboratives favorise à la fois la production langagière par les apprenants, la prise de compte (noticing) des lacunes dans leur interlangue, l'obtention de rétroactions correctives ainsi que la modification de leur production (Guichon et Nicolaev, 2009 ; Kim, 2017 ; Swain, 2000, 2005). Afin de maximiser le développement de compétences en L2, les chercheurs se sont penchés sur l'identification des caractéristiques des tâches qui faciliteraient l'interaction (p. ex. le niveau de difficulté, le nombre d'éléments, le mode) [Gilabert, Manchón et Vasylets, 2016 ; Vasylets, Gilabert et Manchón, 2017] et sur les conditions optimales d'exécution de ces dernières (p. ex. le nombre de répétitions, le délai de planification) [García Mayo et Imaz Agirre, 2016 ; Mackey, Ziegler et Bryfonski, 2016]. En plus de cibler les approches et les tâches, les chercheurs se sont aussi intéressés aux opinions des enseignants en exercice et des enseignants en formation afin d'explorer l'adéquation entre cette approche et les contraintes de la salle de classe (East, 2012 ; Hu, 2013 ; McDonough et Chaikitmongkol, 2007). L'enthousiasme qui entoure l'ELBT reflète cependant surtout les perceptions des enseignants alors que peu d'études empiriques ont examiné de près celles des apprenants. L'étude des perceptions de l'utilité de la tâche par les apprenants ainsi que les éléments précis de la tâche qui suscitent leur intérêt s'avère essentielle : la tâche ne peut être significative que « si l'apprenant est conscient de ce à quoi elle sert, de ce qu'elle est de nature à lui apporter » (Coste, 2009, p. 502). De plus, comme le constataient Hood, Elwood et Falout (2009), « empowering students with voice and incorporating their views into our practice may lead to better classroom experiences and learning outcomes » (p. 23).

La présente étude tente donc de mettre en lumière les perceptions qu'ont les apprenants concernant cette approche ainsi que concernant les caractéristiques de quatre types de tâches utilisées dans leur salle de classe durant un cours de français langue étrangère sur une période de 16 semaines dans un contexte mexicain. Les questions qui guident cette étude sont les suivantes :

1. Comment les apprenants de français langue étrangère perçoivent-ils l'enseignement basé sur les tâches?

2. Quels sont les aspects des tâches pédagogiques utilisées en salle de classe qui suscitent l'intérêt des apprenants?

Avant de décrire l'étude en question, nous définirons mieux en détail en quoi consiste l'ELBT et ce que nous entendons par " tâches pédagogiques ». Ensuite, nous nous tournerons vers les études, en nombre limité, qui ont porté sur les perceptions des apprenants à l'égard de l'ELBT. 


\section{L'ELBT}

L'utilisation de tâches en enseignement des L2s retient l'attention tant des enseignants que des chercheurs (Bygate, 2015 ; Gass et Mackey, 2007 ; Kim, 2015, 2017 ; Van den Branden, 2016). Dans la littérature anglo-saxonne, on trouve une distinction entre l'ELBT (task-based language teaching) et l'enseignement des langues appuyé par les tâches (ELAT, task-supported language teaching) [Bygate, 2015 ; Ellis, 2003, 2017 ; Long, 2015]. Dans le premier cas, les tâches déterminent le contenu du programme éducatif, ce qui signifie qu'à la suite d'une analyse des besoins des apprenants, les tâches choisies s'alignent avec les besoins authentiques des apprenants. Leurs besoins dictent ainsi le choix des tâches (Long, 2015). Dans le second cas, les tâches jouent un rôle important, mais servent plutôt d'outil d'enseignement dans un cours axé sur la communication et l'enseignement des structures linguistiques (p. ex. un plan de cours synthétique) [Long et Crookes, 1992]. Peu importe la façon d'incorporer les tâches dans un cours de langue (ELAT ou ELBT, approches comprises sous ELBT dans ce qui suit), la tâche joue, dans tous les cas, un rôle central dans la salle de classe.

Plusieurs définitions de la tâche ont été proposées depuis le travail séminal de Long (1985; voir Van den Branden, 2016, pour une discussion exhaustive). Long (2015) a proposé une définition très large de la tâche : «tasks are real-world activities people think of when planning, conducting, or recalling their day » (p. 6). Cette définition pourrait ainsi inclure un projet d'aménagement d'une pièce de la maison. Il précise donc qu'une tâche pédagogique est celle qui se réalise dans le contexte d'un cours, mais qui simule des situations du monde réel (p. ex. organiser une fête surprise, classer le meilleur film/restaurant afin de proposer une sortie entre amis). Selon Nunan (2004), la tâche comporte des activités en salle de classe où l'apprenant est amené à utiliser, comprendre, manipuler et interagir dans la langue cible. L'attention des apprenants porte sur le sens plutôt que sur la forme. Nunan a proposé que la tâche pédagogique consiste en une tâche de répétition (rehearsal task) ou une tâche d'activation (activation task). La première «bears a clear and obvious relationship to its corresponding real-world counterpart $»$ (p. 20), mais la deuxième sert plutôt à activer une variété de fonctions et de structures langagières (p. ex. faire des suggestions, être en accord ou désaccord). Il a souligné cependant que les apprenants sont libres d'utiliser " any linguistic means at their disposal to complete the task» (p. 21). Ellis (2003), à son tour, a indiqué qu' une tâche doit respecter quatre critères : (a) la tâche doit mettre l'accent sur le sens, (b) la tâche doit obliger les interlocuteurs à échanger des informations ou des opinions afin de combler une lacune, (c) l'apprenant doit exécuter la tâche en utilisant ses ressources linguistiques et non-linguistiques et (d) l'exécution de la tâche doit promouvoir l'utilisation de la langue cible et avoir un résultat identifiable. En respectant ces critères proposés par Ellis (2003), nous sommes en mesure de développer des tâches dites « authentiques » puisqu'elles reflètent des activités du monde réel. Chaque tâche a pour but de permettre à l'apprenant d'utiliser ses connaissances langagières dans des contextes communicatifs. Les apprenants sont encouragés à produire et, par conséquent, à développer un langage plus avancé et plus complexe, tel qu'on le retrouverait hors de la salle de classe (Ellis, 2003 ; Guichon, 2006 ; Long, 2015 ; Samuda et Bygate, 2008). Dans la présente étude, nous nous appuyons de la définition d'Ellis (2003) puisque celle-ci souligne l'importance de la création d'un environnement propice à l'échange d'informations et d'idées entre les interlocuteurs dans le but de combler une lacune dans ces informations. 
Les enseignants et les chercheurs s'appuyant sur l'ELBT ont recours à une variété de tâches pédagogiques, entre autres, la tâche de type échange d'information/d'opinion (information/opinion gap), jeu de rôle, historiette (story completion), dictogloss, reconstruction de texte (Ellis, 2017). Ellis (2003) a proposé un cadre afin de développer des tâches qui précisent le(s) type(s) de support (input, p. ex. textes écrits et/ou images) ; les conditions (p. ex. façons de présenter les informations) ; la procédure (p. ex. travail individuel, dyade, groupe) et les résultats (p. ex. les produits et processus cognitifs). Mangenot et Soubrié (2010) ont développé un système de classification pour des tâches multimédia qui regroupe des descripteurs spécifiques et non-spécifiques à la tâche. Les descripteurs propres à la tâche précisent notamment le contexte social et les caractéristiques des activités langagières. Récemment, Ellis (2017) a développé une typologie qui classe les tâches pédagogiques selon deux plans : tâche focalisée/non focalisée (focussed/unfocussed) et tâche avec support langagier (input-based) ou tâche favorisant l'extrant langagier (output-based). Une tâche focalisée cible une structure linguistique en particulier mais qui n'est pas explicitement décrite dans la consigne de la tâche. Par exemple, alors qu'ils travaillent à une tâche de type échange d'information, les apprenants sont obligés d'échanger des informations en amont de faire un choix. L'échange d'informations les mènerait à produire des questions (où, combien, quel/quelle, etc.). Une tâche non focalisée peut mener à l'utilisation d'une forme linguistique en particulier mais la tâche n'a pas été créée pour éliciter des formes linguistiques précises. La deuxième dimension se rapporte au produit de la tâche. Une tâche avec support langagier ne requiert aucune production orale ou écrite, mais elle aura un résultat identifiable (p. ex. placer des meubles sur un plan, faire un dessin). Une tâche favorisant l'extrant langagier requiert que l'apprenant produise un texte oral, écrit ou multimodal afin d'atteindre son objectif communicatif. La tâche avec support langagier semble plus adéquate pour des apprenants débutants (Ellis, 2017).

Dans le but de développer des tâches authentiques qui rejoignent les attentes et les préférences des apprenants, il est important de chercher à comprendre les perceptions des apprenants concernant l'approche et plus précisément, les critères des tâches qu'ils préfèrent. En ciblant mieux leurs intérêts, il devient ainsi possible de maintenir leur attention et leur motivation (Bao et Du, 2015 ; Bao et Kirkebæk, 2013). Ainsi dans cette étude, nous nous sommes intéressés aux perceptions des apprenants, soit aux impressions des apprenants concernant les diverses dimensions des tâches réalisées en salle de classe (Brown, 2009 ; Wesely, 2012). Dans le cadre de l'ELBT, le point de vue des apprenants est malheureusement peu documenté ; ce qu'il nous a été possible de recenser est présenté dans la prochaine section. Les études portant sur l'approche communicative en enseignement des langues (communicative language teaching [CLT]) n'ont pas été incluses dans ce recensement des écrits, bien qu'elles offrent des idées convergentes à ce sujet (p. ex. Chung et Huang, 2009 ; Miller, 1995).

\section{L'ELBT et les perceptions des apprenants}

Afin de déterminer les avantages et les limites de l'approche ELBT, les chercheurs se sont d'abord intéressés aux perceptions des enseignants et des enseignants en formation et ce, principalement en Asie-Pacifique (Adams et Newton, 2009 ; Butler, 2005 ; Carless, 2007 ; East, 2012 ; Hu, 2013 ; Jeon, 2006 ; Littlewood, 2007 ; McDonough et Chaikitmongkol, 2007 ; Nishino, 2008) ; en Europe (Mangenot, 2003 ; McAllister, Narcy- 
Combes et Starkey-Perret, 2012 ; Ollivier, 2007, 2010) et en Amérique (Chacón, 2012 ; Douglas et Kim, 2014). Ces études montrent que malgré le fait que les enseignants sont plus habitués à une approche traditionnelle, l'ELBT est généralement bien reçu. Certaines faiblesses ont néanmoins été identifiées, comme l'usage excessif de la langue maternelle (L1) lors des interactions entre les apprenants (Carless, 2002, 2004), la très grande variabilité des productions en langue cible (Butler, 2011 ; Carless, 2002, 2004) ainsi que des contraintes institutionnelles et conceptuelles (Butler, 2011). La salle de classe est un lieu qui rassemble l'enseignant et ses apprenants. Ainsi, nous partons de la prémisse qu'il est important de tenir compte de la perspective des apprenants puisque si les attentes des enseignants et des apprenants ne se rejoignent pas, cela pourrait créer un écart entre l'enseignement et l'apprentissage (Brown, 2009).

De la même manière que les études explorant la perception des enseignants, un nombre important d'études visant la perception des apprenants ont été réalisées avec des apprenants d'anglais langue étrangère en Asie et les résultats semblent indiquer une ouverture des apprenants de cette approche. Ainsi, une étude réalisée par McDonough et Chaikitmongkol (2007) a examiné la réaction des enseignants ainsi que celle des apprenants de l'approche ELBT dans un contexte thaillandais. Les auteurs ont eu recours à un questionnaire pour recueillir les réactions des apprenants à la suite de l'exécution des tâches. Les résultats indiquent que l'utilisation de cette approche a incité les apprenants à prendre plus de responsabilités dans leur apprentissage. Plus précisément, ils ont essayé de trouver des réponses à leurs questions sans compter sur l'aide de l'enseignant. Ils ont également eu recours à des stratégies d'apprentissage afin d'apprendre du vocabulaire. De plus, il semblerait que les apprenants soient devenus moins préoccupés par la grammaire et plus intéressés par la découverte des stratégies métacognitives d'apprentissage. Néanmoins, les chercheurs ont identifié le besoin des apprenants en ce qui concerne la présence de consignes et de rétroaction corrective afin de consolider leurs compétences nouvellement acquises. Les résultats de cette étude confirment que l'approche ELBT a été bien reçue. Cependant, les auteurs n'ont pas questionné les apprenants à propos des tâches spécifiques. De leur côté, Hood et coll. (2009) ont examiné les attitudes d'apprenants au Japon concernant l'approche communicative et les cours d'anglais qui s'appuyaient sur les tâches. Ils ont expliqué que les cours étaient fondés sur les principes d'une approche communicative et que les enseignants utilisaient « tasks extensively in the classroom to achieve course goals » (p. 24). Les questions étaient donc orientées vers la perception des apprenants concernant la production orale, le travail en équipe et le rôle des enseignants. Cette vaste étude a recueilli le point de vue de 739 participants et a affirmé la préférence des apprenants pour l'approche ELBT plutôt qu'une approche plus traditionnelle (i.e. enseignement magistral avec l'accent mis sur la grammaire et le travail individuel), puisque moins centrée sur les apprenants. Les apprenants ont ainsi reconnu l'importance de se responsabiliser et de prendre en charge leur apprentissage au lieu d'attribuer cette responsabilité au professeur. Comme dans l'étude de McDonough et Chaikitmongkol (2007), aucune information portant sur la nature des tâches accomplies par les apprenants n'a été partagée. Plus récemment, Kim, Jung et Tracy-Ventura (2017) ont examiné la perception de 27 apprenants d'anglais langue étrangère dans un contexte coréen afin de déterminer des changements d'attitudes de l'approche et les tâches durant les 16 semaines d'un semestre. La première auteure a développé et enseigné le cours durant lequel les apprenants ont réalisé 12 tâches et ont répondu à six questions après avoir effectué chaque tâche. Ces questions portaient sur leurs perceptions de la complexité, de l'émotion, de leur 
niveau de confiance et de plaisir, de leur motivation ainsi que des occasions d'apprentissage. Les résultats quantitatifs de cette étude démontrent que les apprenants sont devenus plus intéressés à suivre un cours de langue utilisant cette approche, mais qu'ils percevaient tout de même les cours traditionnels comme étant plus bénéfiques pour l'apprentissage des formes linguistiques de façon précise. Les chercheures ont également mené une étude de cas auprès d'une étudiante du groupe. Cette analyse a révélé que les perceptions de cette étudiante ont changé positivement concernant les dimensions du niveau de confiance en soi et des occasions d'apprentissage. Cette recherche est importante car elle nous offre des informations détaillées sur les changements de perception tout au long d'un cours. Cependant, les chercheures ont répété des tâches similaires mais les résultats ne nous permettent pas de déterminer quels types de tâches sont perçus positivement par les apprenants.

Si plusieurs études ont été menées auprès d'apprenants de l'anglais, certains auteurs ont visé d'autres populations. Ainsi, Ollivier (2010) a exploré les perceptions d'apprenants de français langue étrangère par le biais de tâches réelles sur le Web 2.0. Dans cette étude, 39 apprenants ont produit des publications en ligne sur Wikipédia, le tout a permis de l'interaction authentique, par écrit, entre apprenants et locuteurs natifs (non-apprenants). Les données, provenant d'une session de réflexion et de questionnaires remplis par les participants, indiquent que le fait d'être publiés et lus par un vrai public a motivé les apprenants. Cette tâche, portant sur un sujet qui leur était familier, leur a donné l'impression d'avoir des connaissances et une certaine expertise. Bao et Kirkebæk (2013) ont étudié les perceptions d'apprenants universitaires de chinois langue étrangère de niveau débutant $(N=9)$, concernant l'utilisation de tâches par le biais d'observations, d'entretiens semi-dirigés et d'une évaluation de cours. Il s'agissait de tâches fermées (closed task), bidirectionnelles (two-way gap), ayant pour but d'augmenter les occasions de négociation entre apprenants. Les apprenants ont indiqué que les tâches ont favorisé la création d'un environnement stimulant d'apprentissage et le développement de la langue cible. De plus, ils ont perçu une augmentation de leur niveau de confiance et ont rapporté avoir surpassé leurs propres attentes en ce qui concerne le développement de la langue cible. Bao et Du (2015) ont examiné les perceptions de 18 apprenants débutants du chinois langue étrangère. Dans cette étude, les auteurs ont développé trois types de tâches (i.e. échange d'informations, dictogloss et jeu de rôle) et leurs données recueillies proviennent principalement de l'observation de la salle de classe et d'une entrevue à la fin du cours. Les auteurs ont révélé que les tâches servent généralement à (a) encourager la participation, (b) créer des opportunités pour la production orale, (c) diminuer l'anxiété et (d) augmenter le degré de satisfaction de l'apprentissage de la langue. Cependant, les participants de ces deux études ont rapporté que la prononciation leur semble avoir été un aspect négligé de ces tâches. De plus, dans l'optique d'améliorer ces cours, les apprenants ont suggéré les modifications suivantes : une implication plus importante de l'enseignant, une meilleure explication des objectifs de l'ELBT et une variété plus importante de types de tâches, entre autres.

De manière générale, ces études indiquent que les apprenants attribuent à cette approche un certain nombre d'avantages. Cependant, elles soulignent aussi qu'ils ne perçoivent pas d'avantages liés au développement de la prononciation et de la précision du langage. Comme on l'a vu, ces études se sont intéressées à la perception des apprenants concernant l'approche ELBT en général et ce, par le biais de questionnaires et d'observations de salles de classe (p. ex. Kim et coll., 2017 ; McDonough et 
Chaikitmongkol, 2007). L'étude que nous allons aborder examine elle aussi la perception des apprenants, mais, elle prend aussi en compte la perception des apprenants vis-à-vis des types de tâches mises d'avant par cette approche. Dans ce qui suit, nous présenterons la méthodologie adoptée pour notre étude, ainsi que les tâches développées et leurs critères.

\section{Méthodologie}

\section{Contexte}

Cette étude a été réalisée dans le cadre d'un cours de français langue étrangère de niveau universitaire au Mexique. L'auteure, une spécialiste en ELBT, a développé le matériel et a aussi enseigné le cours. Afin de minimiser les situations potentielles de coercition, avant le début de la session, les apprenants ont été informés des objectifs de la recherche et du double rôle qu' allait jouer l'enseignante dans leur cours. Les apprenants avaient l'option de changer de section et/ou pouvaient refuser de participer à n'importe quel moment sans aucune conséquence. Le cours s'est échelonné sur 16 semaines et les apprenants étaient en classe les lundi, mercredi et vendredi, pour une durée de 50 minutes.

\section{Participants}

Ce groupe était constitué de 18 apprenants, dont 17 ont accepté de faire partie de la recherche. Seule la perception des 12 apprenants présents à chaque étape de la collecte de données a été retenue pour l'analyse. Le Tableau 1 indique le genre, l'âge et le niveau de compétences à l'oral et à l'écrit en français et en anglais de ces 12 apprenants. Le groupe comprenait sept femmes et cinq hommes dont l'âge moyen était de 19,5 ans (min : 18 et $\max$ : 24). Selon les niveaux établis par le Cadre européen commun de référence pour les langues (Conseil de l'Europe, 2001), les participants inscrits à ce cours de français étaient des utilisateurs élémentaires (A2) et, à la fin du semestre, devaient atteindre le niveau B1, ou utilisateurs indépendants. Les apprenants, tous inscrits dans le même cours, avaient néanmoins des niveaux différents de compétences en français et en anglais. Ces compétences ont été évaluées avec des tests développés dans le cadre de l'étude (pour le français : version modifiée du diplôme des études de la langue française [DELF]; pour l'anglais : version modifiée du test TOEFL). Pour assurer la fiabilité des scores, les tests de compétences à l'oral et à l'écrit ont été évalués par deux locuteurs natifs du français et de l'anglais qui ont utilisé une échelle de 1 (faible) à 10 (fort). Les apprenants ont changé de partenaire tout au long de la session et ont donc, ont eu l'occasion de travailler avec des partenaires ayant différents niveaux de compétences dans la langue cible. 
Tableau 1

Profil des participants

\begin{tabular}{lllll}
\hline Apprenant & Genre & Âge & $\begin{array}{l}\text { Niveau en } \\
\text { français }\end{array}$ & $\begin{array}{l}\text { Niveau en } \\
\text { anglais }\end{array}$ \\
\hline Tatiana & $\mathrm{F}$ & 18 & 2.1 & 4.4 \\
Alexa & $\mathrm{F}$ & 19 & 2.3 & 6.5 \\
Marta & $\mathrm{F}$ & 20 & 3.3 & 7.0 \\
Paco & $\mathrm{H}$ & 21 & 4.5 & 7.3 \\
Marimar & $\mathrm{F}$ & 24 & 5.0 & 7.4 \\
Ismael & $\mathrm{H}$ & 18 & 5.0 & 5.4 \\
Mateo & $\mathrm{H}$ & 19 & 5.4 & 6.0 \\
Alicia & $\mathrm{F}$ & 19 & 6.3 & 3.4 \\
Jasmine & $\mathrm{F}$ & 18 & 6.5 & 2.2 \\
Geraldina & $\mathrm{F}$ & 18 & 8.1 & 6.4 \\
Felip & $\mathrm{H}$ & 19 & 8.1 & 5.3 \\
David & $\mathrm{H}$ & 21 & 8.8 & 7.3 \\
\hline
\end{tabular}

Note. Les noms des participants ont été modifiés pour assurer la confidentialité.

\section{Tâches pédagogiques}

La méthode d'enseignement adoptée dans ce contexte était guidée par les principes de l'ELAT (Bygate, 2015). La professeure-chercheure a adapté le plan de cours synthétique créé par le Département de langue de l'université mexicaine, pour y introduire des tâches pédagogiques qui au départ n'étaient pas présentes dans le syllabus. Quatre types de tâches ont été développées : échange d'informations, historiette, dictogloss et reconstruction de texte. Chacune a été répétée trois fois pendant les trois cycles, suivant le même ordre. Dans un premier temps, le cycle de familiarisation a permis aux apprenants de mieux comprendre les types de tâches et de minimiser les malaises potentiels associés aux enregistrements. Les données ont été collectées lors du premier et du deuxième cycle. Les caractéristiques des quatre types de tâches utilisés dans cette étude sont détaillées dans le Tableau 2. 
Tableau 2

Caractéristiques des tâches réalisées par les apprenants selon Ellis (2003)

\begin{tabular}{|c|c|c|c|c|c|}
\hline & Intrant & Condition & Procédure & Produit & Processus \\
\hline $\begin{array}{l}\text { Échange } \\
\text { d'informations }\end{array}$ & Image et texte & $\begin{array}{l}\text { Informations } \\
\text { divisées }\end{array}$ & $\begin{array}{l}\text { Échange en } \\
\text { dyade } \\
\text { obligatoire }\end{array}$ & $\begin{array}{l}\text { Résolution ouverte ; } \\
\text { Texte descriptif à l'écrit }\end{array}$ & $\begin{array}{l}\text { Échange à l'oral des } \\
\text { informations ; } \\
\text { Évaluation et comparaison des } \\
\text { informations }\end{array}$ \\
\hline Historiette & Image & $\begin{array}{l}\text { Informations } \\
\text { divisées }\end{array}$ & $\begin{array}{l}\text { Échange en } \\
\text { dyade } \\
\text { obligatoire }\end{array}$ & $\begin{array}{l}\text { Résolution fermée ; } \\
\text { Texte narratif à l'écrit }\end{array}$ & $\begin{array}{l}\text { Description à l'oral des } \\
\text { informations ; } \\
\text { Proposition d'un ordre logique } \\
\text { des images }\end{array}$ \\
\hline Dictogloss & $\begin{array}{l}\text { Texte présenté à } \\
\text { l'oral }\end{array}$ & $\begin{array}{l}\text { Informations } \\
\text { divisées : à un sens } \\
\text { (one-way gap) et à } \\
\text { deux sens (two-way } \\
\text { gap) }\end{array}$ & $\begin{array}{l}\text { Travail } \\
\text { individuel ; } \\
\text { Échange en } \\
\text { dyade } \\
\text { obligatoire }\end{array}$ & $\begin{array}{l}\text { Résolution ouverte ; } \\
\text { Texte reconstruit à } \\
\text { l'écrit }\end{array}$ & $\begin{array}{l}\text { Échange d'idées pour } \\
\text { reconstruire le sens du texte }\end{array}$ \\
\hline $\begin{array}{l}\text { Reconstruction } \\
\text { de texte }\end{array}$ & Texte écrit & $\begin{array}{l}\text { Informations } \\
\text { partagées }\end{array}$ & $\begin{array}{l}\text { Aucun } \\
\text { échange }\end{array}$ & $\begin{array}{l}\text { Résolution ouverte } \\
\text { Texte reconstruit à } \\
\text { l'écrit }\end{array}$ & $\begin{array}{l}\text { Exprimer des idées simulant un } \\
\text { contexte réel ; } \\
\text { Réflexion métalinguistique }\end{array}$ \\
\hline
\end{tabular}




\section{Procédure}

Cette étude fait partie d'un projet de recherche plus vaste portant sur le développement des compétences linguistiques en français langue étrangère où l'on a utilisé divers instruments de mesure : enregistrements des interactions produites durant les tâches, post-tests immédiats, questionnaires, examens de compétences linguistiques en français et en anglais ainsi qu'entretiens semi-dirigés (Payant, 2015 ; Payant et Kim, 2017). Dans le cadre de la présente étude qui vise à découvrir les perceptions des apprenants à propos de l'approche basée sur les tâches en général ainsi que de quatre types de tâches en particulier, seuls les détails partagés lors des entretiens ont été analysés.

Chaque apprenant, après le cycle de familiarisation, a été invité à participer à un entretien semi-dirigé après avoir réalisé deux tâches (voir Tableau 3). Les tâches du premier cycle ont été réalisées lors des semaines 5 et 7 de la session et celles du deuxième cycle, lors des semaines 11 et 13 de la session. Les questions pour les trois premiers entretiens ciblaient les impressions des apprenants vis-à-vis des caractéristiques des tâches ainsi que des interactions avec leurs pairs. Des questions additionnelles ont été posées lors du quatrième entretien afin d'explorer leurs perceptions de l'utilisation de la L1 et L2 dans un cours de français langue étrangère (Payant, 2015). En moyenne, les premiers entretiens ont duré une demi-heure et le dernier, une heure.

Tableau 3

Procédure lors des trois cycles de la collecte

\begin{tabular}{|c|c|c|c|}
\hline \multirow[t]{2}{*}{ Tâche } & Cycle familiarisation & Premier cycle & Deuxième cycle \\
\hline & Semaine 3 & Semaine 5 & Semaines 11 \\
\hline $\begin{array}{l}\text { Échange } \\
\text { d'informations }\end{array}$ & Les jeunes aventuriers & Trouver un appartement & Publicité : semaine santé \\
\hline Historiette & Le cirque & Vivre en collocation & La malbouffe \\
\hline Entretien & Aucun entretien & Entretien \#1 & Entretien \#3 \\
\hline \multirow[b]{2}{*}{ Dictogloss } & Semaine 4 & Semaine 7 & Semaine 13 \\
\hline & Le cinéma & Les voyages & Sites de musique illégaux \\
\hline $\begin{array}{l}\text { Reconstruction } \\
\text { de texte }\end{array}$ & La famille & Lieux insolites & $\begin{array}{l}\text { Assistance offerte à } \\
\text { distance }\end{array}$ \\
\hline Entretien & Aucun entretien & Entretien \#2 & Entretien \#4 \\
\hline
\end{tabular}

Les entretiens, après avoir été transcrits mot-à-mot, ont été importés dans le logiciel Atlas.ti, un logiciel de traitement pour des données qualitatives. Chaque entretien a été lu et annoté par la professeure-chercheure. Lors de cette lecture, toutes les annotations portant sur les perceptions vis-à-vis de l'approche en général, les quatre tâches, les dynamiques entre pairs, l'apprentissage et l'utilisation des L1 et L2 ont été codés, menant à la création de plus de 140 codes. Ces premières annotations ont été discutées avec une collègue, ce qui nous a permis de procéder à une deuxième ronde de codage. Les codes ont été regroupés en thématique et ce 
processus a abouti à l'identification de 10 thèmes, soit les suivantes : cours antérieurs, bénéfices pour l'apprentissage, caractéristiques des tâches, habiletés ciblées, préférences, réactions émotives, stratégies, langage, dynamique entre pairs, types d'apprenant. Par exemple, pour la thématique cours antérieurs, nous avons regroupé, entre autres, les codes suivants : cours magistraux, dynamique, professeure, manuel scolaire, activité de grammaire. Pour la thématique bénéfices, nous avons regroupé les codes : prise de conscience, apprentissage, réinvestissement de connaissances, lexique, appuis entre pairs. Pour la thématique caractéristique des tâches, nous avons regroupés les codes : intrant oral, intrant visuel, intrant écrit. Nous avons ensuite associé ceux-ci avec les types de tâches. La fonction éditeur réseau dans le logiciel Atlas.ti a facilité le processus. Dans cette étude, nous présentons les résultats portant sur les thèmes suivants : cours antérieurs et caractéristiques des tâches. Nous nous sommes limités à ces deux dimensions puisqu'elles sont liées aux aspects des tâches.

\section{Résultats}

\section{Impressions générales vis-à-vis de l'ELBT}

La première question de recherche visait à révéler les perceptions des apprenants vis-àvis de l'ELBT. Les apprenants ont confirmé que l'ELBT était quelque chose nouveau pour eux. En effet, 11 des 12 apprenants ont indiqué qu'ils avaient coutume d'effectuer des activités axées sur la grammaire provenant d'un manuel scolaire. Par exemple, Tatiana a décrit une dynamique de classe typique où la professeure présentait une notion provenant du manuel scolaire et allouait du temps en classe pour faire les exercices : «Las que vienen en el libro, que son parte de la gramática. Entonces ella nos enseñaba y hacíamos los ejercicios que vienen abajo » ( Celles qui venaient du livre, qui faisaient partie de la grammaire. Donc, elle nous enseignait et nous faisions les exercices qui suivaient »). Elle a ajouté, «Nos pasaba al pizarrón y ella nos decía una palabra y nosotros la teníamos que conjugar. Pasaba a dos porque la clase era enorme » (« Nous allions au tableau et elle nous disait un mot et nous devions le conjuguer. On passait deux à la fois parce que la classe était énorme »). Ce type d'activité ne crée pas de situations propices à des interactions prolongées et authentiques entre les apprenants, lesquelles sont nécessaires pour le développement d'une langue additionnelle (Kim, 2015). David s'est rappelé que surgissaient parfois des conversations spontanées, mais que les apprenants revenaient toujours aux activités prescrites par le manuel scolaire:

Generalmente, es seguir lo que dice el libro. Vemos ejercicios, platicamos de repente "ah mira, ¿qué opinan?" y empezamos a platicar sobre, no sé, deportes . . pero luego regresamos a los ejercicios (Normalement, c'est suivre ce que dit le livre. Nous voyons des exercices, nous discutons et tout à coup "ah bon, qu'en pensez-vous ?" et nous commençons à jaser à propos de, je ne sais pas, sports . . . Et donc nous nous mettons à parler de sport, mais ensuite nous retournons aux exercices).

Les apprenants ont tous discuté des limites du manuel. Par exemple, Paco explique que l'utilisation prévisible du manuel mène à la distraction. Il a expliqué, en commençant par décrire le cours actuel, que « Con los ejercicios es mucho más interesante. Estoy más en la clase. . . Pero en la otra, sí me podía ir y decía "luego leo el libro”" ("Avec les exercices, c'est beaucoup plus intéressant. Je suis plus présent dans la classe. . . Mais dans l'autre, je pouvais 
partir et je me disais "je lirai le livre plus tard” »). Bien que le manuel scolaire serve d'outil d'apprentissage, il semble devenir une béquille pour l'apprenant et n'offre pas de motivation pour la production langagière.

Les apprenants ont identifié un avantage important à l'approche basée sur les tâches. Ainsi, ils considèrent que cette approche a facilité le travail de groupe, ce qui a eu un impact positif sur leur perception du cours. Plus précisément, neuf apprenants ont souligné que 1'ELBT a influencé de manière positive la dynamique de classe. Par exemple, Marta a ressenti que «El curso pasado no era tan dinámico y no se le hacía tanto como énfasis en eso (hablar) » (« Le cours précédent n'était pas très dynamique et il ne mettait pas tellement d'emphase sur ceci [parler] »). Les apprenants ont remarqué qu'ils se retrouvaient dans des situations de communication authentiques dans ce cours, ce qui leur a permis de sortir de la routine monotone et répétitive. Felip a précisé qu'auparavant, il pensait connaître les attentes de l'enseignant(e), mais que dans ce nouveau contexte, il est devenu curieux de découvrir ce qu'ils allaient faire en salle de classe :

Siento que hay mucha diferencia. Por ejemplo, de irse a sentar y asi de " hoy, toca esta lección " y tú ya tenías en la mente exactamente qué ibas a hacer. En cambio, aquí era " a ver qué vamos a hacer » (Je pense qu'il y a une grande différence. Par exemple, d'aller s'asseoir et de " aujourd'hui tu travailles cette leçon » et tu avais déjà en tête exactement ce qui allait se passer. En échange, ici c'était «voyons voir ce que nous allons faire »).

En somme, les apprenants ont apprécié l'approche basée sur les tâches et ont confirmé leur préférence en comparant cette approche avec leurs expériences d'apprentissage antérieures qui étaient traditionnelles et guidées par un manuel scolaire.

\section{Les perceptions concernant les tâches collaboratives}

En lien avec notre deuxième objectif, les perceptions des apprenants vis-à-vis des types de tâches utilisées dans ce contexte sont détaillées dans les quatre sections suivantes. Pour chaque type de tâche, nous débuterons avec une description qui se rapporte au Tableau 2 et poursuivrons par une discussion des résultats obtenus.

L'historiette. L'objectif communicatif de l'historiette était de produire un texte narratif s'appuyant sur des images (intrant : image). Afin de promouvoir la négociation du sens, chaque apprenant n'avait reçu que la moitié des images (condition : informations divisées) et devait, dans un premier temps, les décrire à leur partenaire sans les dévoiler (procédure : échange obligatoire). En se basant uniquement sur les descriptions échangées oralement, les apprenants devaient ensuite proposer une séquence logique pour l'histoire (produit : situation fermée). Ils pouvaient dès lors dévoiler leurs photos et apporter des changements nécessaires avant de passer à la dernière étape, soit celle de la rédaction en dyade d'une courte histoire (produit : texte narratif). Cette tâche pédagogique, utilisée dans un contexte éducatif, ressemblent aux demandes linguistiques du monde réel: échanger des informations, être du même avis (séquencer les évènements représentés par les images) et raconter une histoire.

Les résultats montrent qu'une majorité des apprenants ont eu de la difficulté lors de la première étape de la tâche, soit celle de la description initiale des images, puisqu'ils n'avaient 
aucun intrant écrit. Ainsi, pour 10 des 12 apprenants, le plus grand défi a été l'activation du vocabulaire correspondant aux images. Comme l'a expliqué Jasmine : "Por el caso del vocabulario que no lo tenía todo y no podía decir qué cosas tenía la habitación, porque no sabía cómo se decían todas las cosas en francés » («C'est à cause du vocabulaire, puisque je ne l'avais pas tout et que je ne pouvais pas dire les choses qu'il y avait dans l'appartement parce que je ne savais pas comment dire toutes les choses en français »). Cette lacune sur le plan du vocabulaire les a forcés à faire des efforts pour arriver à communiquer les détails de leurs images et à construire une histoire intéressante. Alicia a mentionné à cet effet que "Lo tenemos que hablar solitos nosotros y eso nos obliga a hablar y a tratar de escribir lo que más podamos » (« Nous devons le dire par nous-mêmes et cela nous oblige à parler et à essayer d'écrire le plus qu'on peut »).

En plus de faire face à des défis à ce niveau, six apprenants ont mentionné de manière explicite qu'ils ont trouvé le processus de compréhension difficile (processus : description à l'oral). D'après les consignes énoncées (condition : informations divisées), les apprenants ne pouvaient pas dévoiler leurs images, les forçant ainsi à travailler la compréhension orale. Cependant, comme l'a indiqué Geraldina, leurs conceptualisations de l'image grâce à la description produite par leurs pairs ne reflétaient pas toujours l'image véridique : «Él la describía y yo trataba de picture it en mi mente. . . Entonces cuando me lo enseñó dije "pues sí es muy diferente" " ("Il la décrivait et j'essayais d'imaginer l'image dans ma tête . . donc, quand il me l'a montrée, j'ai dit, "bien oui, elle est très différente" »). Nous constatons que l'intrant visuel et divisé a été une source de défis pour ce groupe d'apprenants, surtout lors de la description orale.

Après avoir décrit les images, les apprenants les ont mises en ordre et ont rédigé une courte histoire. Pour créer ce texte narratif, ils devaient aller au-delà d'une simple description des images et inventer une histoire cohérente, proposant également un dialogue entre les personnages. N'ayant accès qu'à un intrant visuel, les apprenants ont eu de la difficultés face à cette tâche d'écriture. Par exemple, Paco a expliqué que «Esa es la más difícil porque ahí sí no hay ningún texto de dónde agarrarte y tienes que inventarlo todo tú » («Celle-ci c'est le plus difficile puisque là oui il n'y a aucun texte sur lequel tu peux t'appuyer et tu dois tout inventer toi-même »).

La production d'un texte narratif requiert une certaine dose d'imagination de la part des apprenants. Dans ce groupe, un peu plus de la moitié des apprenants a apprécié ce côté créatif. Paco a expliqué ceci : «Me gustó armar historias. Me gustó eso de organizar " (J'ai aimé créer des histoires. J'ai aimé ça organiser »). Cependant, trois apprenants n'ont pas apprécié cet aspect. Notamment, Mateo qui a admis l'avoir trouvé long et laborieux:

En la historia, yo tengo que poner de mí para imaginarme una historia, cosa que no me gusta hacer ... Para crear una historia o para pensar en algo lógico o coherente entre una imagen y otra. Me tardo mucho (Parce que pour l'histoire, je dois m'inspirer de moi pour imaginer une histoire, chose que je n'aime pas faire ... Afin de créer une histoire ou pour penser à quelque chose de logique ou de cohérent entre une image et une autre. Ça me prend beaucoup de temps).

Il semblerait que le processus de rédaction, malgré un intrant visuel mais sans support écrit demeure complexe chez l'apprenant. 
Les constats des apprenants montrent cependant que leurs perceptions sont variables. Lors du deuxième cycle, les apprenants ont continué à travailler avec des images sans texte. Cependant, ils n'ont pas éprouvé ces mêmes difficultés. Tous les apprenants ont expliqué qu'ils avaient un vocabulaire plus riche pour le thème de l'activité et une majorité a remarqué que leurs stratégies de communication s'étaient développées depuis le début du cours. Ismael a comparé ses interactions lors des deux cycles et a expliqué qu'il arrivait à mieux se débrouiller, ce qui est une compétence essentielle du monde :

En este momento, es más fácil. Es que vamos adquiriendo más nivel. Aunque no sepas algo, sales de alguna manera. O sea, buscas otro camino que tú sabes (En ce moment, c'est plus facile. C'est que nous sommes en train d'acquérir plus de connaissances. Même si tu ne sais pas quelque chose, tu t'en sors d'une manière ou d'une autre. C'est-à-dire, tu cherches une autre façon de t'en sortir).

Marimar, elle, a davantage aimé la tâche, car elle a remarqué qu'elle faisait des progrès, autant en production qu'en réception à l'oral : "Ves cuanto avanzamos con el vocabulario y yo le entiendo a David lo que está diciendo y me lo estoy imaginando » (« Tu vois à quel point nous progressons avec le vocabulaire et je comprends plus David [son partenaire], ce qu'il dit et j'arrive à me l'imaginer »). Alicia, ayant éprouvé des difficultés lors du premier cycle, souligne qu'elle est arrivée à mieux se faire comprendre et qu'elle a pu respecter les consignes la poussant à s'exprimer dans la langue cible :

Creo que lo pudimos hacer mejor que la primera vez . . Sí describimos bien las fotos, sí me entendió sin enseñar. Y ya rápido pusimos cuál era cual (Je pense que nous avons pu mieux le faire que la première fois . . Oui, nous avons bien décrit les images et oui, il m'a comprise sans qu'on se montre les images. Et puis rapidement nous avons pu les séquencer).

En résumé, cette tâche a imposé un certain niveau de défi tant en production qu'en réception à l'oral : une difficulté directement liée aux dimensions de ce type de tâche (intrant visuel et échange obligatoire). Cependant, les apprenants ont reconnu qu'ils ont été poussés à utiliser la langue cible, à écouter attentivement et à trouver des façons pour mieux se faire comprendre. De plus, ils ont témoigné qu'ils ont eu moins de difficulté dans ce deuxième cycle de tâches, ce qui est devenu plus agréable pour eux. En sommes, nos analyses révèlent que les tâches qui demandent aux apprenants de faire des descriptions et de créer une histoire uniquement à partir d'images demeurent complexes, mais que les apprenants perçoivent cela positivement.

Reconstruction de texte à l'écrit. Ce deuxième type de tâches avait pour objectif de reconstruire un extrait incomplet à l'écrit (intrant : texte à l'écrit). Plus précisément, les temps de verbe étaient omis et donc, ces informations manquantes rendaient le texte incomplet. Parmi les quatre types de tâches, c'était sans doute la moins authentique. Cependant, elle mettait tout de même l'accent sur le sens, obligeant les apprenants à échanger des opinions et leur demandant d'utiliser des ressources linguistiques (processus) débouchant sur un texte communicatif.

De sorte de produire un texte à partir de ce type d'intrant, il était essentiel que les apprenants retracent le sens du texte (condition : information partagée) dont le contexte déterminerait le temps et l'aspect des verbes ainsi que les structures lexicales convenables. Après 
une reconstruction textuelle en collaboration (procédure : aucun échange), ils devaient comparer leur version avec un modèle produit par l'enseignante afin de les aider à identifier des erreurs et à trouver différents moyens d'exprimer une même idée (produit : résolution ouverte).

À l'exception d'un apprenant, les résultats indiquent une uniformité dans les constats : les apprenants ont de loin préféré cette tâche. En plus d'avoir déclaré que cette tâche ressemblait à un jeu où le défi était de trouver une solution linguistique, ils ont ressenti qu'ils exerçaient un certain niveau de contrôle sur la tâche puisque les informations étaient présentées à l'écrit (intrant : texte écrit). David a clarifié de cette façon : «Me gusta esta porque tienes control de las cosas porque está escrito y tú puedes trabajar y no se te escapa nada " ("J'aime celle-là parce que tu es en contrôle des choses parce que c'est écrit et tu peux travailler et rien ne t'échappe »). Les apprenants ont néanmoins trouvé ce type de tâche parmi les plus difficiles parce qu'il leur fallait intégrer les divers temps et aspects de verbes sans avoir accès à un modèle précis de réponse puisqu'une idée pouvait être exprimée de diverses manières (produit : résolution ouverte). Ceci exigeait des connaissances grammaticales et qu'ils reconstruisent le sens du texte pour pouvoir négocier la forme appropriée. Cependant, les propos des apprenants indiquent que cette difficulté a été appréciée puisqu'elle rejoignait des demandes authentiques de communication (processus), un nouvel aspect pour eux. Marta, faisant allusion à des cours antérieurs, s'est expliquée ainsi :

Así es como debe de ser porque cuando hablamos, mezclamos muchos tiempos. Entonces para mí es muy bien, porque siempre como primero trabajamos el passé composé luego el futur luego el ... no sé qué, y nunca los mezclamos (Ça devrait être comme ça parce que lorsqu'on parle, on mélange plusieurs temps. Donc selon moi c'est très bien, parce qu'on travaille toujours comme en premier le passé composé ensuite le futur ensuite le ... je ne sais pas trop et on ne mélange jamais rien).

Presque la totalité des apprenants (neuf sur 11) a affirmé que cette tâche a mené à la prise de conscience de la forme. À ce sujet, Alicia a expliqué ceci : «Siento que estás buscando los errores . . . . O sea, tú mismo estás ayudándote a buscarlos » (« J'ai l'impression que tu es en train de chercher les erreurs . . . C'est-à-dire, toi-même tu t'aides à les chercher »). Jasmine a comparé cette tâche à une rédaction ouverte et a précisé qu'elle est parvenue à mieux identifier ses propres erreurs grâce à cette tâche puisqu'elle a pris le temps de comparer sa version finale à une seconde version complétée par l'enseignante : « Y a diferencia de cuando escribes, muchas veces no te das cuenta. O nos das las correcciones, pero yo no las veo . . no es como que yo la corrijo » («Contrairement à lorsque tu écris, souvent, tu ne remarques pas. Ou, tu nous donnes les corrections, mais je ne les regarde pas . . . ce n'est pas comme si je corrige »). Ce processus qui permet de travailler le sens d'un texte et de comparer sa version du texte à celle proposée par l'enseignant aide, selon cette apprenante, à ne pas répéter ses propres erreurs : «Te ayuda a que tú sola te des cuenta. Y también creo que para que lo leas y al mismo tiempo vayas captando... y ya no cometas esos errores después » («Ça t'aide à t'en rendre compte. Et aussi je pense qu'en lisant et tu cherches à trouver en même temps . . . et donc tu ne répètes pas ces erreurs après »). Si cette tâche a mené à la prise de conscience de la forme linguistique, elle semblerait également avoir aidé à consolider leurs hypothèses vis-à-vis la langue cible. Felip a expliqué: " [N]ecesitan ponerte a corregir y es poner tus conocimientos en papel . . . Entonces yo creo que es de los mejores porque ves en lo que estás mal » («Elles requièrent qu’ on se mette à corriger et ça nous 
permet de démontrer nos connaissances sur papier .... Donc je crois que c'est parmi les meilleures parce que tu vois où tu n'as pas raison »).

Bien que les apprenants aient indiqué une préférence pour cette tâche, ils ont également manifesté une certaine inquiétude à l'effet qu'elle puisse mener à l'apprentissage de formes erronées (produit : résolution ouverte). Ainsi, Marta dit: «Te confunde mucho, o sea puede ser que "ah está bien . . está mal . . no, sí está mal como se escribe" ( "Cette tâche crée beaucoup de confusion, c'est-à-dire c'est possible que "ah ou c'est bien . . . c'est mal . . . non oui c'est mal . . . comment ça s'écrit" »). La tâche instaure également un certain niveau de doute par rapport à leurs propres connaissances antérieures. Comme l'a expliqué Alexa, « Trabajas con otra compañera y ella te dice algo y tú piensas otras cosas. Entonces te hace dudar porque no sabes si ella está bien o está mal» («Tu travailles avec une autre compagne et elle te dit quelque chose et tu penses différemment. Donc, ça te fait douter de toi-même parce que tu ne sais pas si elle a raison ou si elle a tort »). L'analyse des constats des apprenants indique ainsi que la tâche de type reconstruction de texte a été bien reçue comparativement aux trois autres tâches, et cela, malgré son niveau élevé de difficulté. Nous expliquons ceci par le fait que la tâche écrite n'ait pas exigée aux apprenants d'avoir recours à la créativité et la spontanéité comme cela a été le cas pour l'historiette.

Dictogloss. La tâche du type dictogloss demandait aux apprenants de reconstruire le sens d'un texte présenté à l'oral (intrant : oral). Dans un premier temps, les apprenants écoutaient ce texte afin d'en comprendre le sens général (condition : informations divisées, en un sens). Lors de deux écoutes suivantes, ils prenaient des notes détaillées en travail individuel. Ensuite, en dyades, ils combinaient leurs notes afin de produire une transcription finale qui devait reprendre les idées principales du texte (condition : informations divisées, en deux sens). Cette dynamique faisait en sorte que chaque apprenant pouvait contribuer avec ses idées afin de reconstruire le sens du texte (produit : résolution ouverte à l'écrit). Cette tâche servait en effet à engendrer un processus métacognitif puisque les apprenants doivent, en plus d'écouter, prédire et confirmer les informations produites à l'oral (Vandergrift et Goh, 2012). À la toute fin, la transcription de l'enregistrement a été distribuée aux apprenants. Ils devaient comparer les transcriptions et repérer des similitudes/différences entre celles-ci.

Au sein du groupe, cinq apprenants ont apprécié le fait que, contrairement aux autres tâches introduites dans ce cours, le dictogloss jumelait travail individuel et travail collaboratif (procédure : individuel et en dyade). Cette caractéristique est unique à cette tâche. Lorsque les apprenants travaillent individuellement, l'information va dans un sens (one-way gap) puisque toutes les informations proviennent de l'enregistrement. Toutefois, lorsqu'ils travaillent en collaboration, l'information demeure divisée, mais bidirectionnelle. À ce sujet, Felip a remarqué ceci :

Me pareció muy bien porque al principio es concebirlo individualmente y luego ya le ayudas al otro y si no ... puede que tenga cosas que tú no tienes o al revés. Y al momento de juntarlo, ya es lo interesante para ver y corregirle (el texto) (Ça m'a paru très bien parce qu'au départ, c'est de le conceptualiser individuellement et ensuite là tu aides l'autre et sinon ... il se peut que l'autre ait autre chose que tu n'as pas ou l'inverse. Et au moment de regrouper les textes, là c'est la partie intéressante pour voir et le corriger [le texte]). 
Varier la façon de diviser les informations semble être une caractéristique importante du point de vue des apprenants. Une autre dimension importante de cette tâche est la modalité de l'intrant (texte oral). Une majorité des apprenants a apprécié cette tâche puisqu'elle ciblait la compréhension orale, soit une des dimensions langagières les moins travaillées. Comme l'explique Ismael, « Me pareció bastante útil porque es algo que siento que no hemos trabajado mucho . . la comprensión oral » («Elle m'a paru très utile parce c'est quelque chose que je pense que nous n'avons pas travaillé beaucoup, la compréhension orale »). Jasmine a trouvé que ce type de tâche l'a aidée à faire le lien entre l'orthographe et la prononciation : "Es que escribes mejor y te ayuda a la pronunciación. Sí me gustó » («C'est que tu écris mieux et ça t'aide avec la prononciation. Oui, j'ai aimé »).

Une observation rapportée par les apprenants, qui n'a pas directement trait aux caractéristiques des tâches, est liée aux accents des interlocuteurs. Plus précisément pour cette tâche, les enregistrements ont été produits par des locuteurs québécois qui s'expriment avec un accent avec lequel les apprenants n'étaient pas familier, ce qui leur a permis de découvrir cet accent et d'essayer de le comprendre. Marimar a expliqué en ces termes : "Me gustó porque aprendes el acento de una otra persona externa al cual no estamos acostumbradas » («J'ai aimé parce que tu apprends l'accent d'une autre personne auquel nous ne sommes pas habituées »). Geraldina a également expliqué l'importance d'être exposée à des accents autres que celui de la professeure : «Pues a lo mejor te acostumbras a la maestra y le entiendes nada más a la maestra. O el disco también luego es muy fácil » («Bien, peut-être que tu t'habitues à la professeure, tu comprends uniquement ta professeure. Ou le disque aussi il est très facile »). Cette occasion de pouvoir entendre et de décoder un nouvel accent leur a paru favorable et nous semble important à prendre en considération afin d'exposer les apprenants à des accents internationaux.

Les apprenants se sont beaucoup attardés sur cette tâche étant donné sa difficulté, surtout lors du deuxième cycle. Selon eux, celle-ci est due à la complexité de la langue (i.e. formes grammaticales présentes plus avancées) et au débit des locuteurs qui était plus rapide que lors de la tâche du premier cycle. Alexa a expliqué son point de vue ainsi : "Hicimos una antes. Le entendí mejor al texto. Era de la playa. Pero esta se me dificultó más, será por el vocabulario. Yo creo que es mucho la tecnología » (« Nous en avons fait une auparavant. J'ai mieux compris le texte. C'était à propos de la plage. Mais celle-ci, j'ai eu plus de difficulté, peut-être pour le vocabulaire, je pense que ça portait beaucoup sur la technologie »). David, lui, a élaboré comme suit :

Comparado con la otra grabación que hicimos . . . a la de ahorita, esta usaba otro tipo de gramática más compleja, como por ejemplo la voix passive y también por el tipo de vocabulario que era muy especifico de la tecnología (Si on compare l'autre enregistrement que nous avons fait . . . avec celui de maintenant, celui-ci employait une autre sorte de grammaire, plus complexe, comme par exemple la voix passive et le type de vocabulaire était plus relié à la technologie).

Il est important de souligner que selon les apprenants, c'est le contenu et non le type de tâche, qui a contribué à la difficulté de la tâche. Il faut cependant savoir que les thèmes et structures grammaticales étaient semblables à ceux de la reconstruction de texte. La modalité de l'intrant (orale ou écrite) aura peut-être contribué à sa difficulté. D'un point de vue pédagogique, 
il est intéressant de constater que les apprenants valorisent les tâches difficiles, et dans ce cas, une tâche qui vise diverses compétences langagières (réception orale et production écrite).

Échange d'informations. Pour réaliser la tâche de type échange d'informations, les apprenants ont travaillé en dyades et chacun d'eux avait en main des informations écrites toutes différentes mais complémentaires (condition : informations divisées). De plus, chaque fiche d'information comportait des images comme source d'information venant s'ajouter à celles fournies à l'écrit (intrant : écrit et visuel). Lors du premier cycle, les participants recevaient des informations portant sur des appartements montréalais et lors du deuxième, sur des coutumes de divers pays. L'objectif pour les apprenants était d'échanger l'ensemble des informations en main et de sélectionner un seul appartement où ils auraient aimé vivre lors d'un stage à l'étranger ou encore un seul pays qui avait, selon eux, les coutumes et pratiques les plus intéressantes (processus). Afin de faciliter cette prise de décision, les apprenants devaient d'abord partager leurs informations oralement, évaluer et comparer entre eux toutes les informations reçues et enfin faire le choix du meilleur appartement ou du pays. Par la suite, les apprenants devaient justifier par écrit le choix de l'appartement ou créer un dépliant informatif décrivant le pays sélectionné (produit : texte à l'écrit).

Les réponses obtenues lors des entretiens nous ont permis de constater que les apprenants ont apprécié l'authenticité de ce type de tâche ; ils ont notamment trouvé que les objectifs (i.e. échanger leurs informations et faire une sélection) ont créé un contexte véridique pour la production en langue cible. Par exemple, Ismael, en décrivant cette tâche lors du premier cycle, a remarqué qu'il est fréquent pour un jeune de son âge de discuter de logement. Il a également ajouté que ce thème de discussion pourrait lui être utile s'il décidait d'étudier à l'étranger :

Pienso que son mejores las situaciones de la vida real porque es lo que vas a usar ... Y pienso que fue bastante útil porque si algún día viajo a Canadá o a Francia o algún lugar francófono y tengo un roomie, pues tengo que acordar . . . "vi tales lugares, ¿qué te pareció? "y así es algo real . . no es una situación descabellada. (Je pense que les situations de la vie réelle sont meilleures parce que c'est ce que tu vas utiliser ... Et je pense que ce fut très utile parce que si un jour je voyage au Canada ou en France ou dans un endroit francophone et que j'ai un colloque, bien, je dois être d'accord ... « j'ai vu tels lieux, qu'en penses-tu ?» et ceci est quelque chose de réel ... . ce n'est pas une situation irréelle).

Felip, lui, a expliqué que son partenaire et lui ont aimé le processus de sélection : «Creo que lo padre fue decidir de los departamentos y luego lo chistoso fue que ya que llegamos a uno ... "ah no, pero es que ese no tiene dos cuartos. Es es de un cuarto" " ("Je pense que la partie intéressante était de choisir un des appartements et ensuite, l'ironie était que nous en avons choisi un ... 'ah non, mais c'est que celui-là n'a pas deux chambres. Il a une chambre" »). Dans cet extrait, Felip souligne qu'il n'avait pas bien compris les informations fournies par son partenaire, une erreur due à la condition (information divisée) puisqu'ils avaient choisi un appartement avec une seule chambre. Néanmoins, l'activité lui a plu puisque les partenaires ont pu résoudre leur bris de communication et s'entendre sur le choix d'un appartement.

Cependant, il faut souligner que la majorité des apprenants ont trouvé cette tâche moins dynamique que les autres et que quatre l'ont trouvée trop facile. Ce type de tâche, 
selon eux, ne les a pas suffisamment poussés à la créativité langagière : l'intrant à discuter était précisé dans les fiches d'information. Avec ces informations à leur disposition, ils n'avaient qu'à répéter les informations et ont ainsi trouvé le tout facile. Les propos d'Alicia vont dans ce sens : «Muy fácil porque ya tenía la descripción y entonces nada más era decir » (« Très facile parce que j'avais déjà la description et donc il ne fallait que répéter »). Un aspect créatif manquait à cette tâche, comme l'a expliqué Jasmine : "Está bien porque aprendemos el vocabulario y todo pero sí yo creo que sería más de la imaginación. Así practicaríamos más vocabulario que no tenemos escrito » ("C'est bien parce que nous apprenons le vocabulaire et tout mais oui je pense que ça requerrait plus d'imagination. De cette façon nous pratiquerions plus le vocabulaire que nous n'avons pas écrit »). Pour sa part, Mateo a fait remarquer qu'ils ne faisaient que répéter les informations : «Me acuerdo que lo hicimos bastante mecánico, de "cuales son las especialidades culinarias" y nada más repetíamos lo que estaba escrito acá » ("Je me rappelle que nous l'avions fait de façon assez mécanique, de "quelles sont les spécialités culinaires" et nous ne faisions que répéter les informations écrites »). En somme, bien qu'un espace ait été créé (condition : informations divisées) pour discuter de thèmes intéressants et authentiques, les apprenants ont trouvé que le matériel de soutien les encadrait trop et ne les poussait pas suffisamment à la production langagière spontanée.

\section{Discussion}

L'analyse détaillée des entrevues démontre que ces apprenants valorisent l'approche basée sur les tâches. Par le biais de divers types de tâches, la très grande majorité des apprenants a confirmé avoir pu travailler leurs compétences langagières (linguistique et non-linguistique) de manière authentique. Contrairement à leurs expériences d'apprentissage antérieures, la langue ciblée et les activités ne provenaient pas directement du manuel scolaire. Ils se retrouvaient donc à jouer un rôle actif dans leur apprentissage et à être impliqués cognitivement dans le cours. Ces résultats s'alignent avec ceux de recherches antérieures (Bao et Du, 2015 ; Hood et coll., 2009). De plus, ils corroborent ceux des recherches empiriques pour lesquelles les apprenants ont trouvé : (a) le cours plus dynamique puisqu'ils ne savaient pas toujours à quoi s'attendre, chaque tâche apportant de nouveaux défis ; (b) les tâches pratiques/authentiques importantes et (c) les tâches efficaces pour promouvoir la production orale nécessaire et favorisant le développement de la langue cible. Ces atouts de l'approche par tâches rejoignent les résultats de recherches s'étant focalisées sur le point de vue des apprenants (Bao et Du, 2015 ; Bao et Kirkebæk, 2013 ; Hood et coll., 2009 ; Kim et coll., 2017 ; McDonough et Chaikitmongkol, 2007 ; Ollivier, 2010) ainsi que celui des enseignants (Douglas et Kim, 2014 ; Hu, 2013 ; McDonough et Chaikitmongkol, 2007). La correspondance positive entre cette approche et les perspectives des apprenants est importante pour le développement de tâches intéressantes et motivantes.

Notre contribution principale, grâce à cette étude, est d'avoir établi un lien entre les caractéristiques des tâches et les perceptions des apprenants. Nous avons fait ressortir les caractéristiques des tâches qui ont posé des difficultés et celles qui ont attiré l'attention des apprenants. L'historiette, n'offrant que des fiches visuelles, a soulevé des défis sur le plan lexical. Le dictogloss, n'offrant aucun support à l'écrit, a été une source de défis pour la compréhension orale. La tâche de type échange d'informations, offrant toutes les informations à l'écrit, a limité la production langagière et a mené les apprenants à faire une simple relecture des informations. Finalement, la reconstruction de texte, proposant plusieurs solutions possibles, a 
causé un certain malaise puisque les apprenants, focalisé sur la précision linguistique, ont eu peur d'apprendre des formes erronées. Ils ont néanmoins souligné que l'historiette les a poussés à utiliser la langue cible et à trouver des façons de partager leurs idées lorsqu'ils éprouvaient des lacunes lexicales. Cela s'est produit grâce à l'utilisation de fiches visuelles. Ce type de tâche peut donc inciter les apprenants à développer des stratégies de communication fort utiles dans des contextes hors classe, à savoir, dans des situations de communication authentique. À son tour, la tâche de type échange d'informations a forcé les apprenants à échanger des idées et des opinions. Ils ont cependant jugé cette tâche facile, puisque les informations à échanger étaient fournies. Par ailleurs, les apprenants ont trouvé que la reconstruction de texte les a aidés à prendre conscience de la forme tandis que le dictogloss, à travailler l'écoute. Chaque caractéristique des tâches présente ainsi des avantages. En somme, nous proposons que prendre conscience de ces caractéristiques est important lors du recours à ces types de tâches.

Les apprenants ont également indiqué qu'ils avaient particulièrement apprécié la tâche dictogloss laquelle exploite la compréhension orale. Cette dimension demeure négligée en enseignement des L2s et langues étrangères (Vandergrift, 2007) malgré la prépondérance de ce processus dans la vie réelle. En effet comme l'a indiqué Vandergrift (2011), nous accordons plus de $45 \%$ de notre temps dans une journée à la compréhension orale. Ainsi, ce type de tâche est d'autant plus pertinente puisqu'elle invite les apprenants à jouer un rôle actif dans le processus d'écoute. Dans l'étude de Bao et Du (2015), les apprenants ont rapporté que la prononciation était un aspect négligé dans le travail effectué lors des tâches pédagogiques. Dans la présente étude, les apprenants ont souligné que la tâche dictogloss favorisait en fait la prise de conscience et de plus, les a aidés à établir des liens entre l'orthographe et la prononciation. La recherche portant sur la prononciation et l'ELBT est émergente. Elle confirme que cette compétence spécifique qu'est la prononciation peut aussi être ciblée à travers cette approche pédagogique (Gurzynski-Weiss, Long et Solon, 2017).

La tâche de type échange d'informations a souvent été exploitée par les chercheurs dans leurs études (p. ex. Gass, Mackey et Ross-Feldman, 2011 ; Révész, 2011). L'analyse des constats des apprenants suggère que parmi les différents types de tâches, celle-ci ne poussait pas assez les apprenants et qu'elle les encadrait trop. Dans une étude récente, nous avons examiné les synergies entre les tâches et la technologie (Payant et Bright, 2017). Dans cette étude, les apprenants devaient accomplir une tâche de type échange d'informations. Cependant, plutôt que de leur fournir les détails à partager, les apprenants n’ont reçu qu'un gabarit précisant les catégories à investiguer et devaient naviguer sur Internet afin d'identifier eux-mêmes les informations à partager. L'intégration de cette étape les a amenés à se responsabiliser davantage, les obligeant à trouver de l'information pertinente autant qu'intéressante sur le plan personnel (op. cit.). À la lumière des commentaires des apprenants de ces études et des résultats de recherches antérieures (p. ex. Hood et coll., 2009), il serait avantageux de rendre plus souple ce type de tâche en impliquant les apprenants dans la sélection des informations sur lesquels ils porteront leurs décisions.

Afin d'améliorer la mise en œuvre de l'ELBT, des recherches futures pourraient vérifier si les apprenants débutants ainsi que les plus avancés perçoivent les mêmes difficultés pour ces divers types de tâches. Cette étude ne s'étant basée que sur la perception des apprenants, il serait également pertinent de comparer la perception des apprenants à celle des enseignants afin de mieux cerner là où les deux groupes se rejoignent. D'ailleurs, en plus d'examiner les perceptions concernant les divers types de tâches, il serait intéressant d'explorer celles ayant trait à la construction de la complexité (Robinson, 2001). Enfin, pour valider cette approche à long terme, 
une avenue possible serait celle de comparer les points de vue d'apprenants qui sont exposés à l'ELBT depuis un certain temps, afin de voir si la nouveauté ressentie au départ et les avantages associés à cette approche s'estompent avec le temps. Dans cette même perspective, nous souhaitons encourager les enseignants à développer des tâches semblables dans le cadre de leurs cours de langue et à évaluer les avantages et les enjeux que mettent d'avant cette approche basée sur les tâches.

\section{Conclusion}

L'approche basé sur les tâches est au centre de plusieurs discussions et cela, au sein de nombreux contextes d'apprentissage et la recherche montre que dans ces divers contextes et par le biais d'interactions authentiques, les apprenants développent leurs compétences langagières. La présente étude rejoint un nombre croissant d'études qui se focalisent sur la perception des apprenants, une dimension que nous ne pouvons plus ignorer si l'on souhaite examiner de manière critique les avantages et les limites des divers types de tâches. Les résultats de ces études, sembles à ceux de la nôtre et démontrent clairement que les apprenants réagissent bien aux tâches et aux travaux réalisés en collaboration dans ce contexte d'apprentissage. De plus, notre étude, laquelle fait partie d'une recherche plus vaste sur l'ELBT, nous a permis d'identifier les caractéristiques des tâches pédagogiques perçues difficiles tout autant que celles valorisées par les apprenants de français langue étrangère. Nos analyses montrent que les apprenants ont réussi à accomplir les tâches et qu'ils disent que cette approche comporte de nombreux avantages pour leur apprentissage du français. En somme, nous pensons que cette approche, appuyée par des théories courantes en acquisition de la L2, est une approche qui procure aux apprenants beaucoup d'occasions d'utiliser leur langue cible en contexte communicatif ainsi qu'elle favorise des situations d'apprentissage authentiques et dynamiques.

La correspondance devrait être adressée à Caroline Payant.

Courriel : payant.caroline@uqam.ca

\section{Références}

Adams, R. et Newton, J. (2009). TBLT in Asia: Constraints and opportunities. Asia Journal of English Language Teaching, 19(1), 1-17.

Bao, R. et Du, X. (2015). Implementation of task-based language teaching in Chinese as a foreign language: Benefits and challenges. Language, Culture and Curriculum, 28(3), 291-310. doi:10.1080/07908318.2015.1058392

Bao, R. et Kirkebæk, J. M. (2013). Danish students' perceptions of task-based teaching in Chinese. Dans M. J. Kirkebæk, X. Du, et A. Aarup Jensen (dir.), Teaching and learning culture: Negotiating the context (p. 61-78). Rotterdam, Pays-Bas/Boston, MA/Taipei, Taïwan : Springer Science+Business Media.

Brown, A. V. (2009). Students' and teachers' perceptions of effective foreign language teaching: A comparison of ideals. The Modern Language Journal, 93(1), 46-60.

Butler, Y. G. (2005). Comparative perspectives towards communicative activities among elementary school teachers in South Korea, Japan, and Taiwan. Language Teaching Research, 9, 423-446. https://doi.org/10.1191/13621688051r176oa 
Butler, Y. G. (2011). The implementation of communicative and task-based language teaching in the Asia-Pacific region. Annual Review of Applied Linguistics, 31, 36-

57. https://doi.org/10.1017/S0267190511000122

Bygate, M. (2015). Sources, developments and directions of task-based language teaching. Language Learning Journal, 44, 381-400. http://dx.doi.org/10.1080/09571736.2015.1039566

Carless, D. (2002). Implementing task-based learning with young learners. ELT Journal, 56, 389-396. https://doi.org/10.1093/elt/56.4.389

Carless, D. (2004). Issues in teachers' re-interpretation of a task-based innovation in primary schools. TESOL Quarterly, 38, 639-662. doi:10.2307/3588283

Carless, D. (2007). The suitability of task-based approaches for secondary schools: Perspectives from Hong Kong. System, 35, 595-608. https://doi.org/10.1016/j.system.2007.09.003

Chacón, C. T. (2012). Task-based language teaching through film-oriented activities in a teacher education program in Venezuela. Dans A. Shehadeh et C. A. Coombe (dir.), Task-based language teaching in foreign language contexts: Research and implementation (p. 241266). Amsterdam, Pays-Bas : John Benjamins.

Chung, I.-F. et Huang, Y.-C. (2009). The implementation of communicative language teaching: An investigation of students' viewpoints. The Asia-Pacific Education Researcher, 18, 6778.

Coste, D. (2009). Tâche, progression, curriculum. The Canadian Modern Language Review / La Revue canadienne des langues vivantes, 66(4), 499-510.

Conseil de l'Europe. (2001). Cadre européen commun de référence pour les langues : apprendre, enseigner, évaluer. Paris, France : Didier.

Douglas, S. R. et Kim, M. (2014). Task-based language teaching and English for academic purposes: An investigation into instructor perceptions and practice in the Canadian context. TESL Canada Journal, 31, 1. https://doi.org/10.18806/tesl.v31i0.1184

East, M. (2012). Task-based language teaching from the teachers' perspectives: Insights from New Zealand (vol. 3). Amsterdam, Pays-Bas : John Benjamins.

Ellis, R. (2003). Task-based language learning and teaching. Oxford, Royaume-Uni : Oxford University Press.

Ellis, R. (2017). Position paper: Moving task-based language teaching forward. Language Teaching, 50(04), 507-526. https://doi.org/10.1017/S0261444817000179

Gass, S. M. (2015). Comprehensible input and output in classroom interaction. Dans N. Markee (dir.), The handbook of classroom discourse and interaction (p. 182-197). Malden, MA : John Wiley \& Sons. https://doi.org/10.1002/9781118531242.ch11

Gass, S. M. et Mackey, A. (2007). Input, interaction, and output in second language acquisition. Dans B. VanPatten et J. Williams (dir.), Theories in second language acquisition: An introduction (p. 180-206). New York, NY : Routledge.

Gass, S. M., Mackey, A. et Ross-Feldman, L. (2011). Task-based interactions in classroom and laboratory settings. Language Learning, 61, 189-220. doi:10.1111/j.14679922.2011.00646.x

García Mayo, M. D. P. et Imaz Agirre, A. (2016). Task repetition and its impact on EFL children's negotiation of meaning strategies and pair dynamics: An exploratory study. The Language Learning Journal, 44(4), 451-466. ttp://dx.doi.org/10.1080/09571736.2016.1185799 
Gilabert, R., Manchón, R. et Vasylets, L. (2016). Mode in theoretical and empirical TBLT research: Advancing research agendas. Annual Review of Applied Linguistics (ARAL), 36, 117-135. https://doi.org/10.1017/S0267190515000112

Guichon, N. (2006) Langues et TICE. Méthodologie de conception multimédia. Paris, France : Ophrys.

Guichon, N. et Nicolaev, V. (2009). Caractériser des tâches d'apprentissage et évaluer leur impact sur la production orale de L2. Dans Actes du colloque EPAL 2009, Université Stendhal, Grenoble 3. Repéré à http://epal.u-grenoble3.fr/pdf/epal2009-guichonnicolaev.pdf

Gurzynski-Weiss, L., Long, A. et Solon, M. (2017). TBLT and L2 pronunciation: Do the benefits of tasks extend beyond grammar and lexis? Studies in Second Language Acquisition, 39(2), 213-224. doi:10.1017/S0272263117000080

Hood, M., Elwood, J. et Falout, J. (2009). Student attitudes toward task-based language teaching at Japanese universities. Asian Journal of English Language Teaching, 19, 19-47.

$\mathrm{Hu}, \mathrm{R}$. (2013). Task-based language teaching: Responses from Chinese teachers of English. TESL-EJ, 16(4), 1-21.

Jeon, I.-J. (2006). EFL teachers' perceptions of task-based language teaching: With a focus on Korean secondary classroom practice. Asian EFL Journal, 8, 192-206.

Kim, Y. (2015). The role of tasks as vehicles for language learning in classroom interaction. Dans N. Markee (dir.), The handbook of classroom discourse and interaction (p. 163181). Malden, MA : Wiley.

Kim, Y. (2017). Cognitive-interactionist approaches to L2 instruction. Dans S. Loewen et M. Sato (dir.), The Routledge handbook of instructed second language acquisition (p. 126145). New York. NY : Routledge.

Kim, Y., Jung, Y. et Tracy-Ventura, N. (2017). Implementation of a localized task-based course in an EFL context: A study of students' evolving perceptions. TESOL Quarterly, 51(3), 632660. doi:10.1002/tesq.381

Littlewood, W. (2007). Communicative and task-based language teaching in East Asian classrooms. Language Teaching, 40, 243-249. https://doi.org/10.1017/S0261444807004363

Long, M. H. (1985). A role for instruction in second language acquisition: Task-based language teaching. Dans K. Hyltenstam et M. Pienemann (dir.), Modelling and assessing second language acquisition (vol. 18, p. 77-99). Clevedon, Royaume-Uni : Multilingual Matters.

Long, M. (2015). Second language acquisition and task-based language teaching. Malden, MA : Wiley-Blackwell.

Long, M. H. et Crookes, G. (1992). Three approaches to task-based syllabus design. TESOL Quarterly, 26(1), 27. https://doi.org/10.2307/3587368

Mackey, A., Ziegler, N. et Bryfonski, L. (2016). From SLA research on interaction to TBLT materials. Dans B. Tomlinson (dir.), Second language acquisition research and materials development for language learning (p. 103-118). New York, NY : Routledge.

Mangenot, F. (2003). Tâches et coopération dans deux dispositifs universitaires de formation à distance. Apprentissage des Langues et Systèmes d'Information et de Communication, 6(1), 109-125.

Mangenot, F. et Soubrié, T. (2010). Créer une banque de tâches Internet : quels descripteurs pour quelles utilisations ?. Dans A.-L. Foucher, M. Pothier, C. Rodrigues et V. Quanquin (dir.), La tâche comme point focal de l'apprentissage. Actes du 2e colloque international 
Tidilem (Tice et Didactique des Langues Étrangères et Maternelles), Clermont-Ferrand, France.

McAllister, J., Narcy-Combes, M. F. et Starkey-Perret, R. (2012). Language teachers' perceptions of a task-based learning programme in a French University. Dans A. Shehadeh et C. A. Coombe (dir.), Task-based language teaching in foreign language contexts: Research and implementation (p. 313-342). Amsterdam, Pays-Bas : John Benjamins.

McDonough, K. et Chaikitmongkol, W. (2007). Teachers' and learners' reactions to a task-based EFL course in Thailand. TESOL Quarterly, 41, 107-132. doi:10.1002/j.15457249.2007.tb00042.x

Miller, T. (1995). Japanese learners' reactions to communicative English lessons. JALT Journal, $17,31-53$.

Nishino, T. (2008). Japanese secondary school teachers' beliefs and practices regarding communicative language teaching: An exploratory survey. JALT Journal, 30, 27-50.

Nunan, D. (2004). Task-based language teaching. Cambridge, Royaume-Uni : Cambridge University Press.

Ollivier, C. (2007). Ressources internet, wiki et autonomie de l'apprenant. Dans M.-N. Lamy, F. Mangenot et E. Nissen (dir.), Actes du colloque "Échanger pour apprendre en ligne », Grenoble, France.

Ollivier, C. (2010). Écriture collaborative en ligne-Une approche interactionnelle de la production écrite pour des apprenants acteurs sociaux et motivés. Revue française de linguistique appliquée, 15(2), 121-137.

Payant, C. (2015). Plurilingual learners' beliefs and practices toward native and nonnative language mediation during learner-learner interaction. The Canadian Modern Language Review / La Revue canadienne des langues vivantes, 71(2), 1-25. http://dx.doi.org/10.3138/cmlr.2081.1

Payant, C. et Bright, R. (2017). Technology-mediated tasks: Affordances considered from the learners' perspectives. TESOL Journal, 8(4), 791-810. http://dx.doi.org/10.1002/tesj.333

Payant, C. et Kim, Y. (2017). Impact of task modality on collaborative dialogue among plurilingual learners: A classroom-based study. International Journal of Bilingual Education and Bilingualism. http://dx.doi.org/10.1080/13670050.2017.1292999

Révész, A. (2011). Task complexity, focus on L2 constructions, and individual differences: A classroom-based study. Modern Language Journal, 95, 162-181. doi:10.1111/j.15404781.2011.01241.x

Robinson, P. (2001). Task complexity, task difficulty, and task production: Exploring interactions in a componential framework. Applied Linguistics, 22(1), 27-57. https://doi.org/10.1093/applin/22.1.27

Swain, M. (2000). The output hypothesis and beyond: Mediating acquisition through collaborative dialogue. Dans J. Lantolf (dir.), Sociocultural theory and second language learning (p. 97-114). Oxford, Royaume-Uni : Oxford University Press.

Swain, M. (2005). The output hypothesis: Theory and research. Dans E. Hinkel (dir.), Handbook of research in second language teaching and learning (p. 471-484). Mahwah, NJ : Lawrence Erlbaum.

Samuda, V. et Bygate, M. (2008). Tasks in second language learning. New York, NY : Palgrave Macmillan. 
Van den Branden, K. (2016). Task-based language teaching. Dans G. Hall (dir.), The Routledge handbook of English language teaching (p. 238-252). New York, NY : Routledge.

Vandergrift, L. (2007). Recent developments in second and foreign language listening comprehension research. Language Teaching, 40(3), 191. https://doi.org/10.1017/S0261444807004338

Vandergrift, L. (2011). Second language listening: Presage, process, product, and pedagogy. Dans E. Hinkel (dir.), Handbook of research in second language teaching and learning (vol. 2, p. 455-471). New York, NY : Routledge.

Vandergrift, L. et Goh, C. C. M. (2012). Teaching and learning second language listening: Metacognition in action. New York, NY : Routledge.

Vasylets, O., Gilabert, R. et Manchón, R. M. (2017). The effects of mode and task complexity on second language production. Language Learning, 67, 394-430. doi:10.1111/lang. 12228

Wesely, P. M. (2012). Learner attitudes, perceptions, and beliefs in language learning. Foreign Language Annals, 45(s1), S98-S117. doi:10.111/j.1944-9720.2012.01181.x 